\title{
EDITORIAL
}

\section{Focus on paediatrics 2018}

\author{
Hari Krishnan Kanthimathinathan ${ }^{1^{*}}$ (D) and M. J. Peters ${ }^{2,3}$
}

C 2018 Springer-Verlag GmbH Germany, part of Springer Nature and ESICM

\section{Introduction}

"Medicine is a science of uncertainty and an art of probability" said William Osler. Nowhere is this uncertainty more evident than in paediatric intensive care (PIC) because of the scarcity of evidence in this population. Knowledge gaps exist even among the most commonly used interventions, such as oxygen therapy, mechanical ventilation and cardiovascular support, and common scenarios, such as sepsis [1]. In this article, we review progress made towards reducing these fundamental uncertainties in the last year or so.

\section{Oxygen therapy}

Pulse oximetry is a vital part of intensive care monitoring in children and, yet, no trials have compared saturation targets. Consensus guidelines on mechanical ventilation in critically ill children recommend target $\mathrm{SpO}_{2} 92-97 \%$ when PEEP $<10 \mathrm{cmH}_{2} \mathrm{O}$ and $88-92 \%$ when PEEP $\geq 10 \mathrm{cmH}_{2} \mathrm{O}$ in paediatric acute respiratory distress syndrome (pARDS) [2]. However, there is a lack of data about the safety of oxygen therapy. Since then, Oxy-PICU investigators have reported the results of a pilot randomised trial comparing conservative (88-92\%) versus liberal (>94\%) $\mathrm{SpO}_{2}$ targets in ventilated children receiving supplemental oxygen [3]. The results showed that a conservative oxygen target appeared safe and that a definitive trial was feasible. A full trial is awaited.

\section{High-flow nasal cannula therapy (HFNC)}

Due to ease-of-use and good tolerability, heated humidified high-flow nasal cannula therapy (HFNC) therapy has emerged as a common mode of respiratory support. Despite the widespread use, the exact role of HFNC is

\footnotetext{
${ }^{*}$ Correspondence: dr.h.krishnan@gmail.com

1 Paediatric Intensive Care Unit and KIDS, Birmingham Women's and Children's NHS Foundation Trust, Birmingham, UK

Full author information is available at the end of the article
}

unclear. The TRAMONTANE study showed that HFNC had a higher failure rate than nasal continuous positive airway pressure (nCPAP) [4]. More recently, a randomised trial of HFNC versus standard oxygen therapy in infants with bronchiolitis treated outside the paediatric intensive care unit (PICU) setting showed that HFNC was associated with a significantly lower rate of treatment failure [5]. Despite the results in favour of HFNC, more than three-quarters of infants managed with standard oxygen therapy did not need further escalation of care.

In the PICU setting, HFNC is used more widely and not just in bronchiolitis. There are no outcome data showing superiority of HFNC or CPAP over any other intervention [2]. It is perhaps timely then that the UK Paediatric Intensive Care Society Study Group investigators have reported the feasibility of such a multicentre randomised trial comparing HFNC and nCPAP in both step-up and step-down patients in PICU [6].

\section{Blood pressure targets}

Blood pressure (BP) monitoring and interventions to restore normotension are key management priorities in PICU. Significant uncertainties related to BP monitoring and treatment targets include the following: use of unvalidated algorithms in oscillometric devices, lack of consensus about threshold/definition of hypotension, differences between data from mathematical modelling and observational values, and a lack of trial evidence [7].

Literature published recently illustrates the uncertainties well. Using non-invasive BP readings in children admitted to PICU, Abdelrazeq et al. found that the 50th centile for systolic BP in young children admitted to PICU was higher than the US NIH taskforce values [8]. Ray et al. reported that non-invasive BP readings provided a systematically higher systolic, lower diastolic and mean BP values than the invasive readings [9]. It is therefore unclear as to which BP should be used to target therapies. Technological advances, further observational

\section{Springer}


data, clinical trials and consensus are all needed to minimise some of these troubling uncertainties.

\section{Paediatric early warning scores (PEWS)}

A safe and effective system of care needs tools to recognise, respond and escalate support in deteriorating children. Paediatric early warning scores (PEWS)-based assessment is thought to reduce the variability in this process. The EPOCH cluster randomised clinical trial compared the introduction of the 'bedside PEWS' to usual care in hospitalised children [10]. This large and impressive study showed that 'bedside PEWS' was not associated with a reduction in mortality. However, the overall mortality rate was less than $0.2 \%$.

What implications does the EPOCH trial have for the future of early warning systems? Given the complexities

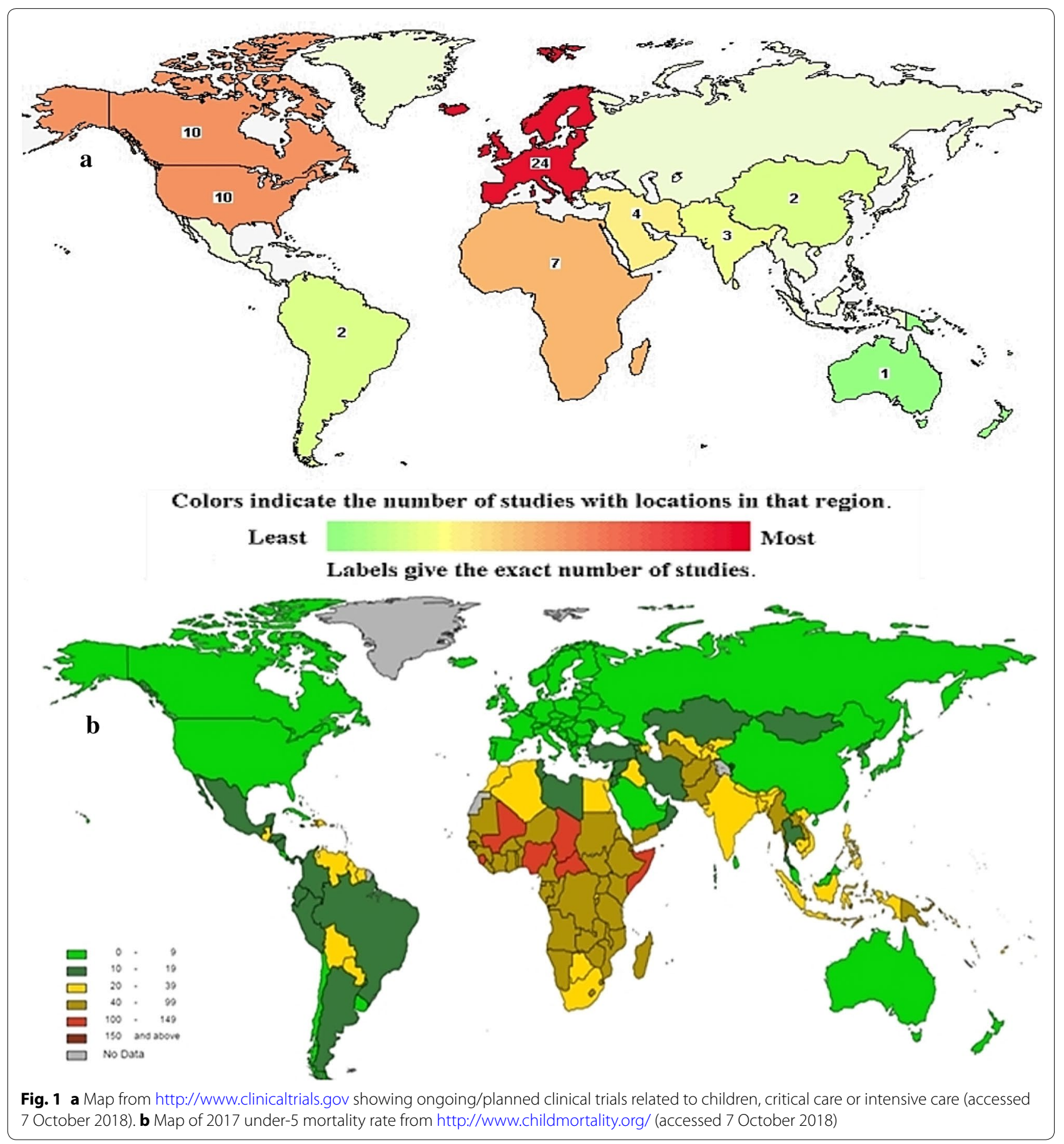


of why children die and the relatively small proportion of preventable deaths in children, this study was probably under-powered, despite the massive numbers studied, to detect a mortality difference [11]. In contrast, this study provides robust evidence that PEWS is not harmful. PEWS or similar systems are here to stay.

\section{Sepsis}

Sepsis-related mortality remains a key issue in PICU. Schlapbach et al. analysed data from a cohort of paediatric sepsis patients in PICU to predict mortality from variables available within $1 \mathrm{~h}$ of admission. They observed a mortality rate of $8.5 \%$, with nearly half the deaths occurring within the first $48 \mathrm{~h}$ of PICU admission [12].

Revised definitions for sepsis based on the SOFA score (sequential organ failure assessment) as the marker of organ dysfunction were recently published for adults. While efforts are ongoing for a similar revision in paediatrics, the lack of consensus on the paediatric version of the SOFA score may be an issue. Schlapbach et al. derived an age-adapted SOFA score [13], in which the prognostic accuracy of the age-adapted SOFA and PELOD-2 scores performed better than SIRS and qSOFA scores for paediatric sepsis-related mortality. Other proposals for a paediatric SOFA score have also been published $[14,15]$. Given the significance of a new universally applicable paediatric SOFA score, Kawasaki et al. suggested that the way forward requires worldwide collaboration of paediatric intensivists [16]. A renewed focus on re-evaluating sepsis epidemiology with novel and generalisable sepsis stratification tools is necessary to design efficient trials to improve care for paediatric sepsis [17].

\section{Paediatric acute respiratory distress syndrome}

In pARDS, it is unclear what is the optimum positive endexpiratory pressure (PEEP) value, how to assess optimal PEEP and whether markers of oxygenation or compliance predict best PEEP [2]. Khemani et al. observed that children managed with lower PEEP relative to $\mathrm{FiO}_{2}$ than that recommended by the 'ARDSNet model' in pARDS had a higher mortality than those who had PEEP in line with or higher [18]. The need for a clinical trial is evident.

However, pARDS is a heterogeneous entity and the most appropriate study design should be adopted. Yehya et al. observed that children with direct and indirect ARDS had distinct clinical characteristics, but similar outcomes [19]. In contrast, they observed that infectious and non-infectious ARDS demonstrated heterogeneity of clinical characteristics, mortality, and predictors of mortality. The identification of different pARDS phenotypes has significant implications for study design. De Luca et al. have pointed out the pros and cons of 'lumping' (pragmatic, more inclusive) and 'splitting' (explanatory, less inclusive) patients of different phenotypes in a clinical trial [20]. Both types of clinical trial evidence are required.

\section{Summary}

Recent efforts to establish large-scale collaborative paediatric critical care research networks across the world have meant that several clinical trials are in progress or planned. It is noteworthy, however, that there is a significant disparity between geographical regions where clinical trials are ongoing and those where a large number of childhood deaths occur (Fig. 1). North America and Europe, despite their relatively low childhood mortality, continue to initiate the majority of PIC trials. However, it is heartening that trials are being performed in other parts of the world where evidence is most needed and there is the most to gain from improving outcomes: Africa, the Indian sub-continent, Latin America and the Middle-East. This is despite significant challenges related to priorities, funding, and support for research within the developing countries.

Significant inroads have been made towards reducing fundamental uncertainties for paediatric intensivists in many fronts. Osler said "See, and then reason and compare and control". And that is what we, as researchers and clinicians, must continue to do.

\section{Author details \\ 'Paediatric Intensive Care Unit and KIDS, Birmingham Women's and Children's NHS Foundation Trust, Birmingham, UK. ${ }^{2}$ Respiratory, Critical Care and Anaes- thesia Unit, University College London Great Ormond Street, Institute of Child Health, London, UK. ${ }^{3}$ Paediatric Intensive Care Unit, Great Ormond St Hospital NHS Foundation Trust, London, UK. \\ Funding \\ This work received no direct funding but was supported by the National Insti- tute for Health Research, Great Ormond Street Hospital Biomedical Research Centre. The views expressed are those of the authors and not necessarily those of the NHS, the NIHR or the UK Department of Health.}

Received: 8 October 2018 Accepted: 15 October 2018

Published online: 23 October 2018

\section{References}

1. Peters MJ, Argent A, Festa M et al (2017) The intensive care medicine clinical research agenda in paediatrics. Intensive Care Med 43:1210-1224

2. Kneyber MCJ, de Luca D, Calderini E et al (2017) Recommendations for mechanical ventilation of critically ill children from the Paediatric Mechanical Ventilation Consensus Conference (PEMVECC). Intensive Care Med 43:1764-1780

3. Peters MJ, Jones GAL, Wiley D et al (2018) Conservative versus liberal oxygenation targets in critically ill children: the randomised multiplecentre pilot Oxy-PICU trial. Intensive Care Med 44:1240-1248. https://doi. org/10.1007/s00134-018-5232-7

4. Milési C, Essouri S, Pouyau R et al (2017) High flow nasal cannula (HFNC) versus nasal continuous positive airway pressure (nCPAP) for the initial 
respiratory management of acute viral bronchiolitis in young infants: a multicenter randomized controlled trial (TRAMONTANE study). Intensive Care Med 43:209-216. https://doi.org/10.1007/s00134-016-4617-8

5. Franklin D, Babl FE, Schlapbach LJ et al (2018) A randomized trial of high-flow oxygen therapy in infants with bronchiolitis. N Engl J Med 378:1121-1131. https://doi.org/10.1056/NEJMoa1714855

6. Ramnarayan P, Lister P, Dominguez T et al (2018) FIRST-line support for assistance in breathing in children (FIRST-ABC): a multicentre pilot randomised controlled trial of high-flow nasal cannula therapy versus continuous positive airway pressure in paediatric critical care. Crit Care 22:144. https://doi.org/10.1186/s13054-018-2080-3

7. Marlais M, Lyttle MD, Inwald D (2017) Ten concerns about blood pressure measurement and targets in paediatric sepsis. Intensive Care Med 43:433-435. https://doi.org/10.1007/s00134-016-4642-7

8. Abdelrazeq S, Ray S, Rogers L et al (2018) Age-associated blood pressure distributions in paediatric intensive care units differ from healthy children. Intensive Care Med 44:384-386

9. Ray S, Rogers L, Noren DP et al (2017) Risk of over-diagnosis of hypotension in children: a comparative analysis of over 50,000 blood pressure measurements. Intensive Care Med 43:1540-1541

10. Parshuram CS, Dryden-Palmer K, Farrell C et al (2018) Effect of a pediatric early warning system on all-cause mortality in hospitalized pediatric patients: the EPOCH randomized clinical trial. JAMA 319:1002-1012

11. Chapman SM, Wray J, Oulton K, Peters MJ (2018) "Death is not the answer": the challenge of measuring the impact of early warning systems. Arch Dis Child. https://doi.org/10.1136/archdischild-2018-315392

12. Schlapbach LJ, MacLaren G, Festa M et al (2017) Prediction of pediatric sepsis mortality within $1 \mathrm{~h}$ of intensive care admission. Intensive Care Med 43:1085-1096
13. Schlapbach LJ, Straney L, Bellomo R et al (2018) Prognostic accuracy of age-adapted SOFA, SIRS, PELOD-2, and qSOFA for in-hospital mortality among children with suspected infection admitted to the intensive care unit. Intensive Care Med 44:179-188

14. Shime N, Kawasaki T, Nakagawa S (2017) Proposal of a new pediatric sequential organ failure assessment score for possible validation. Pediatr Crit Care Med 18:98-99. https://doi.org/10.1097/PCC.0000000000001009

15. Matics TJ, Sanchez-Pinto LN (2017) Adaptation and validation of a pediatric sequential organ failure assessment score and evaluation of the Sepsis-3 definitions in critically ill children. JAMA Pediatr. https://doi. org/10.1001/jamapediatrics.2017.2352

16. Kawasaki T, Shime N, Straney L et al (2018) Paediatric sequential organ failure assessment score (pSOFA): a plea for the world-wide collaboration for consensus. Intensive Care Med 44:995-997

17. Schlapbach LJ, Javouhey E, Jansen NJG (2017) Paediatric sepsis: old wine in new bottles? Intensive Care Med 43:1686-1689. https://doi. org/10.1007/s00134-017-4800-6

18. Khemani RG, Parvathaneni K, Yehya N et al (2018) Positive end-expiratory pressure lower than the ARDS network protocol is associated with higher pediatric acute respiratory distress syndrome mortality. Am J Respir Crit Care Med 198:77-89. https://doi.org/10.1164/rccm.201707-1404OC

19. Yehya N, Keim G, Thomas NJ (2018) Subtypes of pediatric acute respiratory distress syndrome have different predictors of mortality. Intensive Care Med 44:1230-1239. https://doi.org/10.1007/s00134-018-5286-6

20. De Luca D, Harrison DA, Peters MJ (2018) "Lumping or splitting" in paediatric acute respiratory distress syndrome (PARDS). Intensive Care Med 44:1548-1550 